\title{
ESTUDO COMPARATIVO ENTRE BOBINAS DE CORPO E SUPERFÍCIE NA MAMOGRAFIA POR RESSONÂNCIA MAGNÉTICA DE PRÓTESES DE SILICONE*
}

\author{
Anabel Medeiros Scaranelo ${ }^{1}$
}

Resumo Ressonância magnética de mamas de pacientes com próteses mamárias de silicone foi realizada utilizandose parâmetros técnicos predeterminados, comparando-se bobina de superfície dedicada às mamas e bobina de corpo no mesmo grupo. Foram comparadas 43 próteses mamárias do tipo gel de silicone em 24 pacientes. Observou-se que a relação sinal-ruído obtida pela bobina de superfície foi maior que a obtida pela bobina de corpo. Dobras do elastômero foram identificadas com igual resolução pelas bobinas de superfície e de corpo em cerca de $82 \%$ à direita e $95 \%$ à esquerda. Apenas em cerca de $5 \%$ dos casos as dobras foram visibilizadas utilizando-se exclusivamente a bobina de superfície específica para mamas. Demonstrou-se que o sinal do "linguine" encontrado nas próteses é visto pelos dois métodos praticamente igual. Houve apenas um caso em que o sinal do "linguine" foi visto pela bobina de superfície e não pela bobina de corpo. Evidenciou-se também que boceladuras ou deformidade de contornos podem ser avaliadas com alta concordância, utilizando-se tanto a bobina de superfície quanto a bobina de corpo. Concluiu-se que, embora a qualidade da imagem seja melhor com o uso da bobina de superfície, próteses mamárias também poderão ser avaliadas pela bobina de corpo na ausência de uma bobina específica para mamas, com o mesmo resultado diagnóstico.

Unitermos: Bobina de corpo. Bobina de superfície. Ressonância magnética. Próteses mamárias. Silicone.

\begin{abstract}
Comparative study between body and surface coils in magnetic resonance mammography of silicone prosthesis.

Magnetic resonance imaging scans using predefined parameters were performed in patients with silicone breast implants. The same group of patients was submitted to magnetic resonance imaging scans using surface breast coils and body coils, and the results were compared. A total of $\mathbf{4 3}$ single-lumen silicone-gel breast implants in 24 patients were examined. The signal-to-noise ratio was greater for the breast coil than for the body coil. Radial folds were identified with equal resolution by both coils in almost $82 \%$ of the cases on the right side and $95 \%$ on the left side. In about $5 \%$ of the cases the folds were seen exclusively when the breast coil was used. The "linguine sign" was identified almost equally with both methods. In just one case the "linguine sign" was observed only by using the breast coil. Identification of bulging or irregularity of contours were concordant using both techniques. We concluded that although magnetic resonance imaging quality is better using a dedicated breast coil, silicone breast implants can be assessed with the same diagnostic accuracy using a body coil.

Key words: Body coil. Dedicated breast coil. Magnetic resonance imaging. Breast implants. Silicone.
\end{abstract}

\section{INTRODUÇÃO}

As próteses mamárias de silicone ${ }^{(\mathbf{1})}$ têm sido amplamente utilizadas, tanto na cirurgia plástica estética como na reparadora (reconstrução mamária após mastectomia), desde o início dos anos 60.

* Trabalho realizado no Laboratório Fleury e no Departamento de Diagnóstico por Imagem da Universidade Federal de São Paulo/Escola Paulista de Medicina (Unifesp/EPM), São Paulo, SP.

1. Membro Titular do Colégio Brasileiro de Radiologia Mestre em Radiologia Clínica e Doutora em Medicina pela Unifesp/EPM, Médica Assistente Doutora do Departamento de Diagnóstico por Imagem da Unifesp/EPM.

Endereço para correspondência: Dra. Anabel Medeiros Scaranelo. Avenida Jacutinga, 343, apto. 43, Moema. São Paulo, SP, 04530-015.E-mail: anabelms@uol.com.br

Aceito para publicação em 18/10/2000.
Quanto maior a idade da prótese (tempo de inclusão no organismo), maior o risco de ocorrer sua ruptura ${ }^{(2)}$. Devido ao risco de ruptura e à possibilidade das complicações existentes (dor localizada, parestesias, possibilidade de associação com doença auto-imune generalizada, deslocamento da prótese, contratura capsular e alterações no seu contorno), nos Estados Unidos as próteses de silicone do tipo gel não estão mais autorizadas pelo Federal Drug Administration para uso em cirurgia estética desde abril de 1992.

O diagnóstico de certeza de provável ruptura de prótese de silicone pelo exame físico geralmente é difícil, prin- cipalmente nas rupturas intracapsulares. Os sinais e sintomas de uma prótese rota geralmente são bastante $\operatorname{vagos}^{(3)}$.

Os radiologistas são freqüentemente solicitados para avaliar, por meio de métodos de imagem, as próteses mamárias de silicone. Um método de diagnóstico por imagem sensível e específico para pacientes com próteses de silicone é necessário, a fim de se detectar, precocemente, sinais de alterações e poder-se planejar a cirurgia a ser realizada.

A ressonância magnética (RM), como método de imagem para avaliação das mamas com próteses, foi introduzida há menos de uma década ${ }^{(4)}$. Grande parcela dos equipamentos disponíveis 
em nosso meio não possui bobina dedicada à mama, considerada acessório e tendo custo adicional.

Este estudo comparativo avalia a bobina de corpo (BC) e a bobina de superfície (BS) dedicada às mamas, analisando imagens adquiridas no mesmo equipamento, no mesmo momento.

\section{MATERIAL E MÉTODO}

Foram estudadas 43 próteses mamárias de silicone por meio de RM, num período de quatro meses, correspondendo a 24 pacientes encaminhadas ao setor de imagem do Laboratório Fleury, São Paulo, SP, independentemente do fato de possuírem alterações clínicas nas suas próteses mamárias. Não foi usado em nenhum exame qualquer tipo de sedação ou anestesia.

Sete regiões de interesse (ROI) foram analisadas por meio de cursores colocados nas imagens, a fim de se estudar a relação sinal/ruído (S/R) (Figura 1). O ROI número 1 foi colocado fora da imagem, para mensurar o ruído.

$\mathrm{Na}$ mama direita foram colocados os ROIs números 2, 3 e 4 e na mama esquerda, os ROIs números 5, 6 e 7 . Os ROIs números 2, 4, 5 e 7 foram colocados em pontos simétricos sobre a linha hemiaxilar e na região retroareolar, sobre o parênquima glandular. Os ROIs números 3 e 6 foram colocados na porção central da prótese de silicone.
Posteriormente, foram agrupados em três regiões, R1, R2 e R3, respectivamente quadrantes laterais (ROIs 2 e 5), próteses (ROIs 3 e 6) e parênquima retroareolar (ROIs 4 e 7), e divididos esses valores pelo ROI 1 para se encontrar a relação $\mathrm{S} / \mathrm{R}$ de cada região.

Os contornos das próteses (elastômero + cápsula fibrosa) foram classificados em regulares e irregulares, conforme a evidência de boceladuras, calcificações da cápsula (margens serrilhadas) ou solução de continuidade (Figura 2).

$\mathrm{O}$ interior das próteses (camada de gel de silicone) foi classificado em homogêneo e heterogêneo, conforme a evidência de dobras do envelope de silicone ou de sua fragmentação, representando o sinal do "linguine" (Figuras 3 e 4).

Os exames foram realizados em um equipamento da marca GE (General Electric Medical Systems, Milwaukee), modelo Signa Advantage (Figura 5), "software" V. 5.4 com magneto supercondutor de $0,5 \mathrm{~T}$, com gradientes de campo de 10 $\mathrm{mT} / \mathrm{m}$. As bobinas utilizadas foram com geometria em sela - BC - e com geometria em quadratura - BS - para mamas, conforme especificação do construtor (Figura 6).

Previamente, antes de iniciar este trabalho, realizou-se um estudo piloto adquirindo imagens com tempos de repetição de $2.500,4.000$ e 5.500 ms e tempo de eco efetivo calculado pelo próprio aparelho, na mesma paciente. Os tempos de $4.000 \mathrm{~ms}$ para o TR e de 170 $\mathrm{ms}$ para o TE foram escolhidos pelo fato de tornar o silicone brilhante, contrastando com o parênquima mamário e tecido adiposo adjacente, que ficam hipointensos (cinzas) na seqüência "fast spin-echo" (FSE)-T2.

Outros parâmetros técnicos, tais como matriz de aquisição de $256 \times 256$ com duas medidas, espessura de corte e FOV, foram escolhidos segundo dados da li-

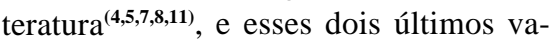
riando conforme a anatomia da paciente e as dimensões da prótese mamária.

Os seguintes parâmetros técnicos foram utilizados: seqüência de FSE-T2 ( $\mathrm{TR}=4.000 \mathrm{~ms} / \mathrm{TE}=170 \mathrm{~ms})$, matriz de $256 \times 256$, espessura de cortes variando de 3,0 a $5,0 \mathrm{~mm}$ com incremento de 1,0 a $1,5 \mathrm{~mm}$, número de medidas igual a 2 , FOV de 18 a $28 \mathrm{~cm}$ nas próteses unilaterais e de 32 a $36 \mathrm{~cm}$ quando bilateral, gradiente de fase ântero-posterior, com saturação colocada posteriormente, e banda de $6,4 \mathrm{kHz}$. Não foi administrado meio de contraste paramagnético nesse estudo. $\mathrm{O}$ uso de acoplamento cardíaco e/ou respiratório também não foi empregado, devido ao fato de aumentar o tempo de aquisição.

A posição em decúbito ventral foi utilizada em virtude da configuração da BS (Figura 6) adaptada para esta posição e também por produzir imagem de boa qualidade com menos artefatos de movimentos respiratórios.

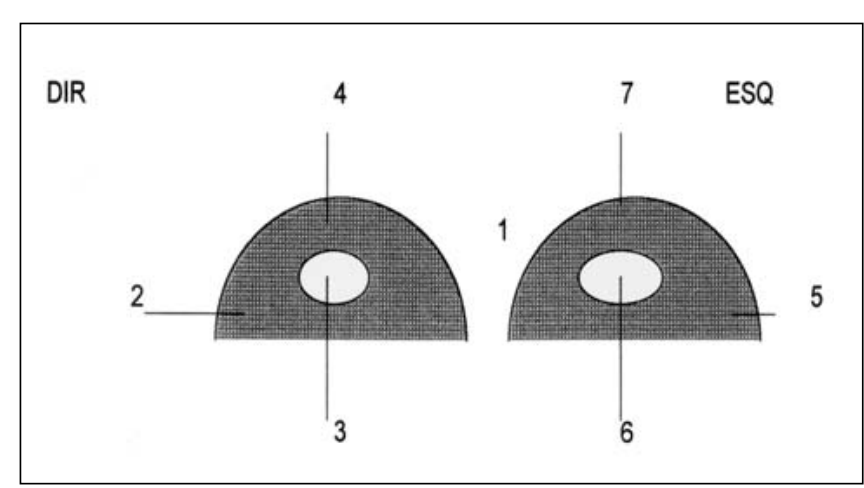

Figura 1. Representação espacial do local onde foi mensurada a intensidade de sinal.

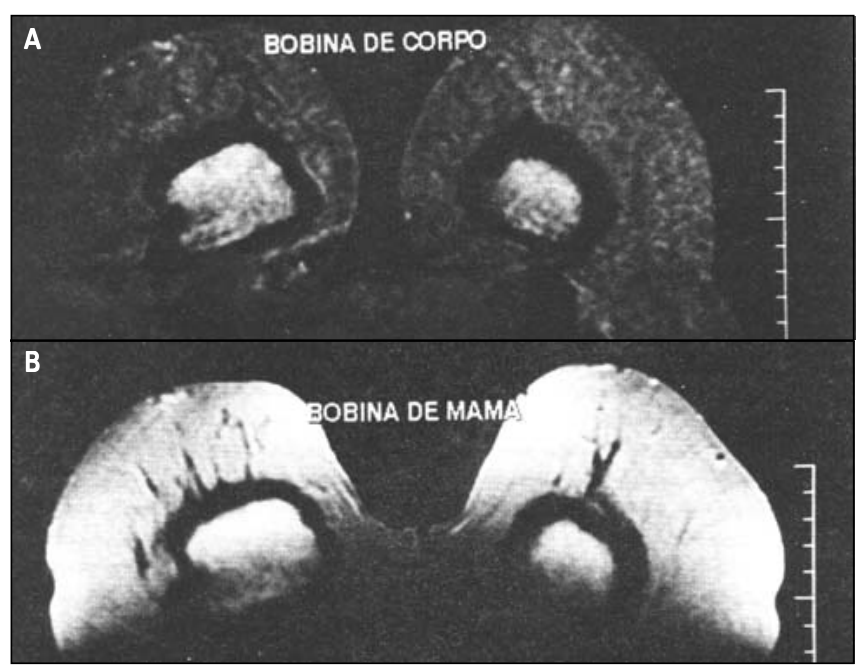

Figura 2. Imagens adquiridas com bobina de corpo (A) e bobina de superfície (B). Ambas mostram contorno irregular devido a importante calcificação da cápsula fibrosa (prótese colocada há 20 anos). 


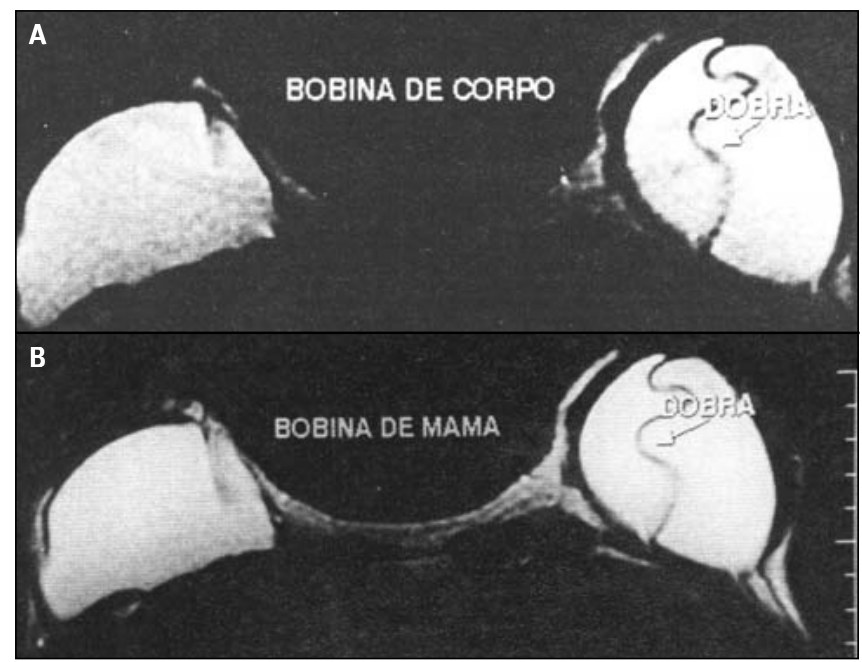

Figura 3. Dobras em ambas as próteses, que se estendem de um extremo a outro. Imagem ruidosa mas com boa definição da estrutura, adquirida com bobina de corpo (A). Mesmo caso, com imagem adquirida com bobina de mama (B).

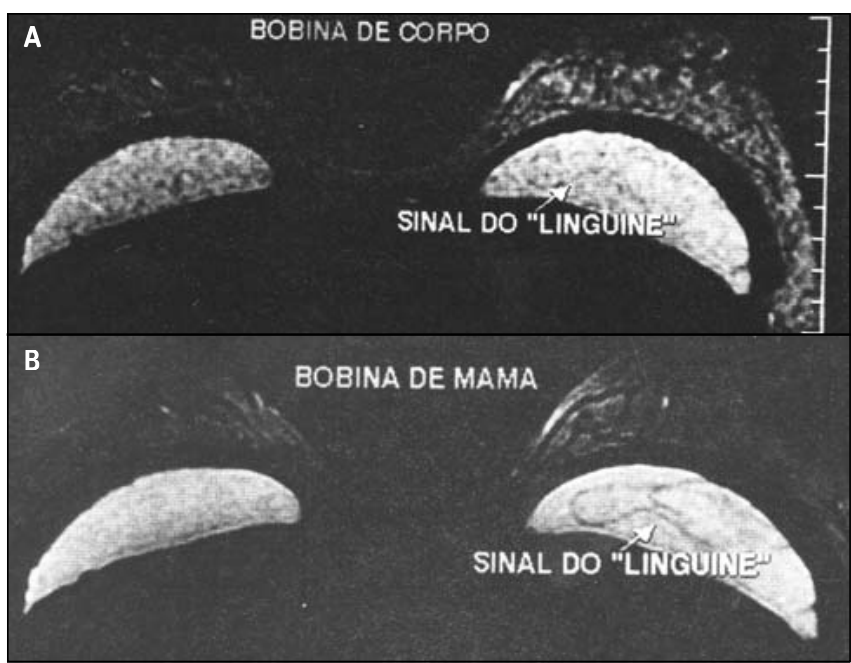

Figura 4. Ruptura intracapsular. Múltiplas linhas curvilíneas de hipossinal correspondendo ao elastômero colapsado são visibilizadas no interior da prótese direita, tanto com o uso da bobina de corpo (A) quanto da de superfície (B). Prótese esquerda normal. Este aspecto, que lembra fios de macarrão, foi chamado de sinal do "linguine".

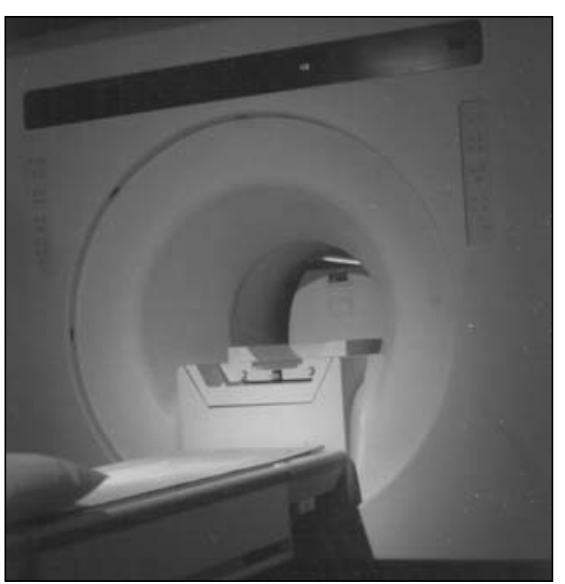

Figura 5. Equipamento de ressonância magnética Signa Advantage de 0,5 T, com bobina de corpo utilizada.

Para a análise dos resultados, utilizou-se o teste de McNemar, com o objetivo de comparar, para cada exame, os resultados observados com o uso de BC e de BS.

Tendo em vista a necessidade de se avaliar as unidades prótese-mama em função da localização direita ou esquerda, aplicou-se também o teste de Wilcoxon para certificar-se da independência da lateralidade.

Em todos os testes fixou-se em 0,05 ou $5 \%(\alpha \leq 0,05)$ o nível para a rejeição da prótese da hipótese de nulidade, assinalando-se com um asterisco os valores significantes.

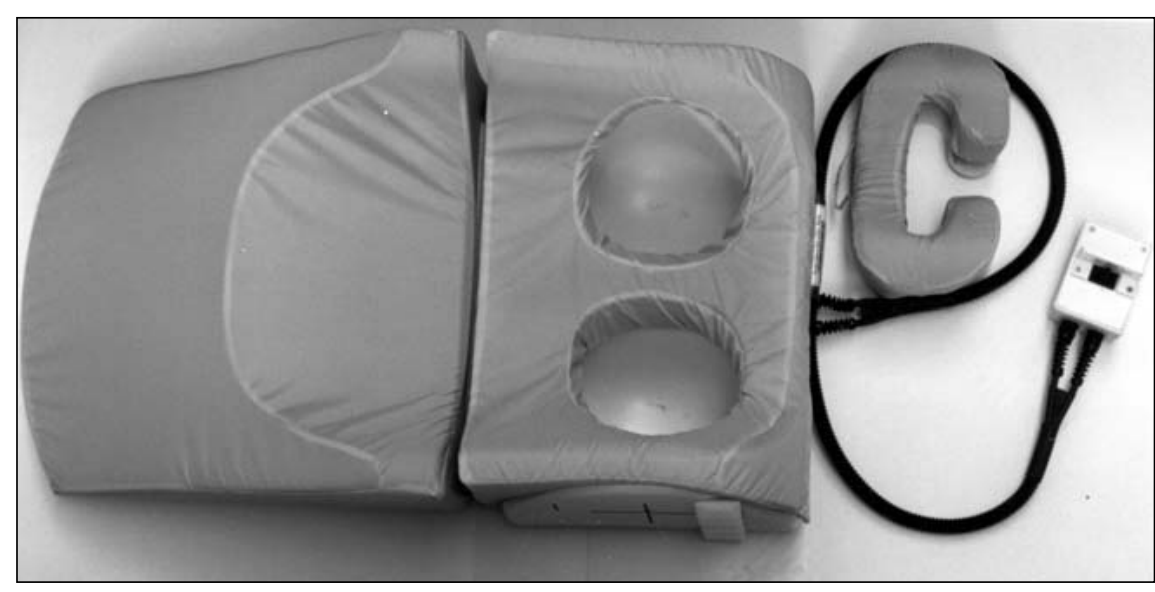

Figura 6. Bobina de superfície dedicada a mama utilizada (Breast coil, GE Medical System, Milwaukee). Notar a conformação anatômica para acomodação das mamas em decúbito ventral.

\section{RESULTADOS}

A relação $\mathrm{S} / \mathrm{R}$ da região $\mathrm{R} 2$ (prótese) nas pacientes com próteses bilaterais, quando se comparou o lado direito com o lado esquerdo em função da BS, foi estatisticamente significante, e a relação $\mathrm{S} / \mathrm{R}$ das regiões $\mathrm{R} 1$ e R3 não. Com a $\mathrm{BC}$, os resultados nas três regiões não foram estatisticamente significantes (Tabelas $1,2$ e 3$)$.

Nas regiões R1 e R2 direita, a relação S/R foi maior pela BS em $90,91 \%$ da amostra. Na região R3 direita, cinco casos $(22,82 \%)$ tiveram relação $S / R$ maior pela BC (Tabelas 4, 5 e 6).
Nas regiões R1 e R2 esquerda, a relação S/R foi maior pela BS em $90,48 \%$ da amostra. Poucos casos apresentaram relação $\mathrm{S} / \mathrm{R}$ da BS menor que a da BC (9,52\%). Na região R3 esquerda, 33,33\% tiveram relação $S / R$ da $B S$ menor que a da BC (Tabelas 7, 8 e 9).

Alterações de contorno das próteses da mama direita (boceladuras, calcificações da cápsula) foram vistas com a BS e BC, com concordância de $81,8 \%$, e das próteses da mama esquerda com $90,47 \%$. Em $18,18 \%$ do lado direito as alterações foram detectadas apenas pela BS, não sendo caracterizadas pela $\mathrm{BC}$ em $9,5 \%$ para o lado esquerdo (Tabelas 10 e 11). 
Tabela 1 Relação $S / R$, segundo a região $R 1$ analisada em função dos lados direito e esquerdo, detectada pela BS e pela BC, em pacientes com próteses bilaterais.

\begin{tabular}{|c|c|c|c|c|}
\hline \multirow{2}{*}{ Paciente } & \multicolumn{2}{|c|}{ BS } & \multicolumn{2}{|c|}{$B C$} \\
\hline & Dir. & Esq. & Dir. & Esq. \\
\hline 1 & 119,00 & 125,00 & 28,38 & 85,00 \\
\hline 2 & 143,00 & 142,00 & 38,11 & 132,00 \\
\hline 3 & 39,50 & 40,00 & 24,38 & 20,35 \\
\hline 4 & 38,00 & 38,33 & 36,44 & 36,11 \\
\hline 5 & 13,60 & 13,00 & 4,54 & 3,06 \\
\hline 6 & 17,67 & 16,67 & 9,74 & 15,16 \\
\hline 7 & 17,67 & 22,00 & 5,75 & 27,90 \\
\hline 8 & 6,88 & 10,00 & 5,84 & 2,98 \\
\hline 9 & 24,70 & 43,40 & 5,89 & 17,30 \\
\hline 10 & 16,00 & 19,00 & 5,20 & 4,28 \\
\hline 11 & 4,00 & 8,67 & 3,84 & 7,46 \\
\hline 12 & 18,80 & 17,00 & 11,36 & 12,19 \\
\hline 13 & 17,50 & 8,75 & 1,57 & 3,05 \\
\hline 14 & 81,00 & 64,00 & 13,60 & 11,06 \\
\hline 15 & 9,66 & 8,66 & 13,50 & 0,56 \\
\hline 16 & 9,75 & 9,00 & 6,82 & 0,91 \\
\hline 17 & 10,75 & 13,00 & 4,89 & 5,25 \\
\hline 18 & 12,30 & 11,10 & 15,87 & 2,15 \\
\hline 19 & 36,90 & 35,27 & 9,81 & 7,91 \\
\hline Média & 33,51 & 33,94 & 12,92 & 21,30 \\
\hline Mediana & 17,67 & 17,00 & 9,74 & 11,06 \\
\hline & $\begin{array}{l}T \text { cal } \\
T \text { crit }\end{array}$ & $\begin{array}{l}\text { ste d } \\
00 ;\end{array}$ & $\mathrm{T}$ & $\begin{array}{l}88,00 ; \\
46,00 \text {. }\end{array}$ \\
\hline
\end{tabular}

A presença de dobras do elastômero foi detectada em $72,72 \%$ das próteses na mama direita pela BS e em $54,54 \%$ utilizando-se a BC, com concordância de $81,81 \%$ (Tabela 12). Não houve nenhum caso detectado pela BC isoladamente que não pudesse ser identificado pela BS. Em 18,18\% somente puderam ser identificadas com a utilização da bobina dedicada à mama.

Dobras do elastômero das próteses da mama esquerda foram identificadas pela BS em $61,9 \%$ das próteses e pela $\mathrm{BC}$ em $57,14 \%$. Também não houve nenhum caso detectado pela $\mathrm{BC}$ isoladamente que não pudesse ser identificado pela BS. Em apenas um caso $(4,76 \%)$ foram identificadas dobras pela BS que não foram vistas pela BC (Tabela 13).

A presença do sinal de "linguine" foi detectada igualmente pela BS e pela BC, obtendo-se concordância de $100 \%$ na mama direita. Na mama esquerda, um
Tabela 2 Relação $S / R$, segundo a região $R 2$ analisada em função dos lados direito e esquerdo, detectada pela $\mathrm{BS}$ e pela $\mathrm{BC}$, em pacientes com próteses bilaterais.

\begin{tabular}{|c|r|r|r|r|}
\hline \multirow{2}{*}{ Paciente } & \multicolumn{2}{|c|}{ BS } & \multicolumn{2}{c|}{ BC } \\
\cline { 2 - 5 } & Dir. & Esq. & \multicolumn{1}{|c|}{ Dir. } & Esq. \\
\hline 1 & 135,00 & 141,00 & 26,08 & 86,38 \\
2 & 156,00 & 165,00 & 45,33 & 149,22 \\
3 & 43,50 & 48,50 & 16,53 & 17,85 \\
4 & 46,67 & 52,00 & 45,67 & 50,11 \\
5 & 18,80 & 20,40 & 4,54 & 3,51 \\
6 & 25,33 & 30,67 & 23,84 & 23,68 \\
7 & 26,67 & 28,67 & 13,10 & 32,00 \\
8 & 14,63 & 16,50 & 13,92 & 14,18 \\
9 & 24,50 & 79,30 & 8,70 & 30,41 \\
10 & 19,50 & 20,75 & 4,15 & 5,78 \\
11 & 13,00 & 14,00 & 12,97 & 10,27 \\
12 & 34,00 & 38,60 & 19,51 & 20,36 \\
13 & 24,50 & 21,25 & 4,10 & 9,57 \\
14 & 77,00 & 74,00 & 20,93 & 19,46 \\
15 & 33,33 & 34,66 & 2,22 & 0,72 \\
16 & 19,50 & 19,00 & 10,11 & 8,97 \\
17 & 25,75 & 26,50 & 8,72 & 8,27 \\
18 & 17,30 & 20,90 & 23,00 & 23,54 \\
19 & 47,81 & 47,72 & 16,03 & 11,31 \\
\hline Média & 42,25 & 47,34 & 16,81 & 27,66 \\
Mediana & 25,75 & 30,67 & 13,92 & 17,85 \\
\hline & & Teste de Wilcoxon: \\
& T calc. = 24,00*; & T calc. $=62,00 ;$ \\
& T crit. = 46,00. & T crit. $=46,00$. \\
\hline
\end{tabular}

caso em 21 analisados não foi concordante, estatisticamente não-significante (Tabelas 14 e 15).

\section{DISCUSSÃO}

A utilização da $\mathrm{BC}$ na realização de RM para o estudo de próteses mamárias de silicone vinha sendo aplicada até o desenvolvimento tecnológico de bobinas dedicadas para mamas.

Não há relatos, nos últimos anos, se a $\mathrm{BC}$, presente na totalidade dos equipamentos de RM comprados para uso geral, é eficaz na detecção de alterações de próteses de silicone quando comparada com uma bobina feita especificamente para mamas, mas com um custo econômico adicional significativo na sua aquisição como acessório.

Sinha et al. ${ }^{(6)}$ utilizaram uma adaptação de BS para ombro para a avaliação de ruptura de prótese de silicone. Gor-
Tabela 3 Relação S/R, segundo a região $R 3$ analisada em função dos lados direito e esquerdo, detectada pela $B S$ e pela $B C$, em pacientes com próteses bilaterais.

\begin{tabular}{|c|r|r|r|r|}
\hline \multirow{2}{*}{ Paciente } & \multicolumn{2}{|c|}{ BS } & \multicolumn{2}{c|}{ BC } \\
\cline { 2 - 5 } & Dir. & Esq. & Dir. & Esq. \\
\hline 1 & 79,00 & 48,00 & 12,23 & 40,77 \\
2 & 41,00 & 12,00 & 13,89 & 26,00 \\
3 & 28,50 & 32,50 & 14,50 & 20,76 \\
4 & 55,33 & 55,00 & 30,44 & 22,56 \\
5 & 9,60 & 11,00 & 5,86 & 6,42 \\
6 & 9,67 & 7,00 & 4,95 & 1,37 \\
7 & 8,33 & 15,67 & 1,35 & 25,60 \\
8 & 9,00 & 8,13 & 6,92 & 8,80 \\
9 & 3,20 & 44,70 & 5,15 & 10,70 \\
10 & 9,25 & 10,00 & 2,61 & 2,37 \\
11 & 3,33 & 1,67 & 7,03 & 7,03 \\
12 & 20,60 & 9,60 & 8,95 & 5,90 \\
13 & 6,25 & 4,75 & 1,05 & 1,39 \\
14 & 38,00 & 36,00 & 6,20 & 18,33 \\
15 & 3,00 & 4,00 & 4,13 & 4,38 \\
16 & 4,75 & 17,75 & 4,44 & 8,02 \\
17 & 2,25 & 6,50 & 1,08 & 0,57 \\
18 & 3,20 & 1,90 & 4,96 & 3,48 \\
19 & 34,09 & 35,36 & 7,63 & 8,50 \\
\hline Média & 19,39 & 19,03 & 7,55 & 11,70 \\
Mediana & 9,25 & 11,00 & 11,70 & 8,02 \\
\hline & & Teste de Wilcoxon: \\
& T calc. $=92,00 ;$ & T calc. $=46,00 ;$ \\
& T crit. = 46,00. & T crit. $=40,00$. \\
\hline
\end{tabular}

czyca et al..$^{(5)}$ relataram ter utilizado, inicialmente, BC para a realização de exames de 143 mulheres e criaram uma adaptação em duas BSs para ombro, unindoas como se fossem uma só, a fim de melhorar a relação SR. Ahn et al. ${ }^{(4)}$ também utilizaram-se de BS na maior parte da sua casuística (100 pacientes).

É sabido que a qualidade da imagem é diretamente proporcional ao aumento da relação SR, e que BSs produzem relação SR bem maior que $\mathrm{BCs}^{(5)}$.

O diagnóstico de alteração na prótese de silicone que demande nova intervenção cirúrgica, no caso ruptura, deve ser estabelecido a um custo mínimo.

Outros autores ${ }^{(4,5,7)}$, similarmente a este trabalho, também utilizaram a sequiência FSE com TR e TE idênticos de 4.000/170 ms. Posteriormente, há relatos na literatura da utilização de valores um pouco maiores: TR de 3.500 a 5.700 $\mathrm{ms}$ e TE de 170 a $200 \mathrm{~ms}^{(8-10)}$. 
Tabela 4 Relação S/R das próteses (região R2) na mama direita detectada pela BS e pela $\mathrm{BC}$.

\begin{tabular}{|c|c|c|}
\hline Paciente & BS & $B C$ \\
\hline 1 & 135,00 & 26,08 \\
\hline 2 & 156,00 & 45,33 \\
\hline 3 & 43,50 & 16,53 \\
\hline 4 & 46,67 & 45,67 \\
\hline 5 & 18,80 & 4,54 \\
\hline 6 & 25,33 & 23,84 \\
\hline 7 & 26,67 & 13,10 \\
\hline 8 & 14,63 & 13,92 \\
\hline 9 & 24,50 & 8,70 \\
\hline 10 & 19,50 & 4,15 \\
\hline 11 & 13,00 & 12,97 \\
\hline 12 & 34,00 & 19,51 \\
\hline 13 & 24,50 & 4,10 \\
\hline 14 & 77,00 & 20,93 \\
\hline 15 & 33,33 & 2,22 \\
\hline 16 & 19,50 & 10,11 \\
\hline 17 & 25,75 & 8,72 \\
\hline 18 & 17,30 & 23,00 \\
\hline 19 & 47,81 & 16,03 \\
\hline 20 & 209,00 & 15,48 \\
\hline 21 & 203,00 & 241,33 \\
\hline 22 & 12,47 & 4,86 \\
\hline Média & 55,78 & 26,21 \\
\hline Mediana & 26,41 & 14,70 \\
\hline
\end{tabular}

Optou-se por espessuras abaixo de 5 $\mathrm{mm}$ neste estudo (foram utilizadas de 3 a $5 \mathrm{~mm}$ ), devido ao fato de obter-se melhores relações $\mathrm{SR}^{(\mathbf{6})}$.

$\mathrm{Na}$ casuística, existem 19 pacientes com próteses bilaterais, três com prótese unilateral direita e dois com prótese unilateral esquerda. Estudaram-se as unidades prótese-mama separadamente em função do lado, pois são variáveis independentes. Todos os resultados obtidos, estudando-se separadamente as unidades prótese-mama direita e esquerda em função da BS e BC, foram estatisticamente significantes.

As unidades prótese-mama-lado foram estudadas comparativamente em função de três regiões: R1 ou quadrante lateral, R2 ou prótese e R3 ou região de parênquima retroareolar. Embora a pretensão fosse estudar a prótese, ou seja, a região R2, escolheu-se também analisar
Tabela 5 Relação S/R do quadrante lateral (região R1) na mama direita detectada pela BS e pela BC.

\begin{tabular}{|c|r|r|}
\hline Paciente & BS & BC \\
\hline 1 & 119,00 & 28,38 \\
2 & 143,00 & 38,11 \\
3 & 39,50 & 24,38 \\
4 & 38,00 & 36,44 \\
5 & 13,60 & 4,54 \\
6 & 17,67 & 9,74 \\
7 & 17,67 & 5,75 \\
8 & 6,88 & 5,84 \\
9 & 24,70 & 5,89 \\
10 & 16,00 & 5,20 \\
11 & 4,00 & 3,84 \\
12 & 18,80 & 11,36 \\
13 & 17,50 & 1,57 \\
14 & 81,00 & 13,60 \\
15 & 9,66 & 13,50 \\
16 & 9,75 & 6,82 \\
17 & 10,75 & 4,89 \\
18 & 12,30 & 15,87 \\
19 & 36,90 & 9,81 \\
20 & 163,00 & 12,76 \\
21 & 194,00 & 217,00 \\
22 & 0,91 & 0,42 \\
\hline Média & 45,21 & 21,62 \\
Mediana & 17,67 & 9,77 \\
\hline & Teste de Wilcoxon: \\
& T calc. =30,00* T crit. = 66,00. \\
BS $>$ BC. \\
\\
\end{tabular}

os quadrantes laterais para verificar a homogeneidade ou não do campo magnético, quando se compara o quadrante lateral direito e o quadrante lateral esquerdo nas pacientes com próteses bilaterais.

Nos quadrantes laterais (região R1) da mama direita, a relação $S / R$ foi maior pela BS na maioria das pacientes. Em $9,09 \%$ da amostra tal não ocorreu, embora com todos os parâmetros técnicos idênticos; numa delas ( $\left.\mathrm{n}^{\mathrm{o}} 18\right)$, observaram-se artefatos causados por movimentos respiratórios utilizando-se a bobina de mama.

$\mathrm{Na}$ região $\mathrm{R} 2$ direita, a relação $\mathrm{S} / \mathrm{R}$ também foi maior pela BS na maioria das pacientes, cerca de $91 \%$. O inverso deu-se em dois casos, sendo um coincidente ( $\left.n^{\circ} 18\right)$ com os movimentos respiratórios e outro caso $\left(\mathrm{n}^{\mathrm{o}} 21\right)$ relacionado à mobilização da paciente entre as se-
Tabela 6 Relação S/R da região retro-areolar (região R3) na mama direita detectada pela $\mathrm{BS}$ e pela $\mathrm{BC}$.

\begin{tabular}{|c|c|c|}
\hline Paciente & BS & $B C$ \\
\hline 1 & 79,00 & 12,23 \\
\hline 2 & 41,00 & 13,89 \\
\hline 3 & 28,50 & 14,50 \\
\hline 4 & 55,33 & 30,44 \\
\hline 5 & 9,60 & 5,86 \\
\hline 6 & 9,67 & 4,95 \\
\hline 7 & 8,33 & 1,35 \\
\hline 8 & 9,00 & 6,92 \\
\hline 9 & 3,20 & 5,15 \\
\hline 10 & 9,25 & 2,61 \\
\hline 11 & 3,33 & 7,03 \\
\hline 12 & 20,60 & 8,95 \\
\hline 13 & 6,25 & 1,05 \\
\hline 14 & 38,00 & 6,20 \\
\hline 15 & 3,00 & 4,13 \\
\hline 16 & 4,75 & 4,44 \\
\hline 17 & 2,25 & 1,08 \\
\hline 18 & 3,20 & 4,96 \\
\hline 19 & 34,09 & 7,63 \\
\hline 20 & 89,00 & 8,68 \\
\hline 21 & 206,00 & 238,67 \\
\hline 22 & 0,28 & 0,67 \\
\hline Média & 30,16 & 17,69 \\
\hline Mediana & 9,43 & 6,03 \\
\hline
\end{tabular}

qüências com BS e BC, que acarretou diferenças na topografia.

$\mathrm{Na}$ região do parênquima retroareolar (região R3) da mama direita, cinco pacientes $(22,82 \%)$ apresentaram relação $\mathrm{S} / \mathrm{R}$ maior pela BC. Duas pacientes são coincidentes com a da região $\mathrm{R} 2$, e para as demais $\left(\mathrm{n}^{\mathrm{O}} \mathrm{s} 11,15\right.$ e 22$)$ esses aspectos estão muito provavelmente relacionados a diferenças anatômicas na quantidade de tecido glandular e heterogeneidade deste no local.

Considerando-se o lado esquerdo, a paciente de $\mathrm{n}^{\mathrm{O}} 18$ obteve o mesmo comportamento que foi encontrado para o lado direito nas três regiões. A paciente de $n^{0} 7$ também apresentou relação $S / R$ maior para a BC nas três regiões do lado esquerdo, e como possui prótese bilateral, acreditamos que o campo magnético não fosse homogêneo, pois o mesmo não ocorreu para o lado direito. 
Tabela 7 Relação S/R das próteses (região R2) na mama esquerda detectada pela BS e pela $\mathrm{BC}$.

\begin{tabular}{|c|c|c|}
\hline Paciente & BS & $\mathrm{BC}$ \\
\hline 1 & 141,00 & 86,68 \\
\hline 2 & 165,00 & 149,22 \\
\hline 3 & 48,50 & 17,85 \\
\hline 4 & 52,00 & 50,11 \\
\hline 5 & 20,40 & 3,51 \\
\hline 6 & 30,67 & 23,68 \\
\hline 7 & 28,67 & 32,00 \\
\hline 8 & 16,50 & 14,18 \\
\hline 9 & 79,30 & 30,41 \\
\hline 10 & 20,75 & 5,78 \\
\hline 11 & 14,00 & 10,27 \\
\hline 12 & 38,60 & 20,36 \\
\hline 13 & 21,25 & 9,57 \\
\hline 14 & 74,00 & 19,46 \\
\hline 15 & 34,66 & 0,72 \\
\hline 16 & 19,00 & 8,97 \\
\hline 17 & 26,50 & 8,27 \\
\hline 18 & 20,90 & 23,54 \\
\hline 19 & 47,72 & 11,31 \\
\hline 20 & 109,00 & 56,47 \\
\hline 21 & 23,00 & 15,48 \\
\hline Média & 49,12 & 28,45 \\
\hline Mediana & 30,67 & 17,85 \\
\hline
\end{tabular}

Tabela 10 Pacientes portadoras de próteses na mama direita, segundo a presença ou não de irregularidade de contornos (boceladura, deformidade) detectada pela BC e pela BS.

\begin{tabular}{|l|c|c|c|}
\hline \multirow{2}{*}{ BS } & \multicolumn{2}{|c|}{ BC } & \multirow{2}{*}{ Total } \\
\cline { 2 - 3 } & Regular & Irregular & \\
\hline Regular & 10 & 0 & 10 \\
Irregular & 4 & 8 & 12 \\
\hline Total & 14 & 8 & 22 \\
\hline
\end{tabular}

Teste de McNemar: $p=0,0625$ ou 6,25\%; porcentagem de concordância: $18 / 22=81,81 \%$.

Tabela 13 Pacientes portadoras de próteses na mama esquerda, segundo a presença ou não de dobras do envelope elastomérico detectada pela BC e pela BS.

\begin{tabular}{|c|c|c|c|}
\hline \multirow{2}{*}{ BS } & \multicolumn{2}{|c|}{ BC } & \multirow{2}{*}{ Total } \\
\cline { 2 - 3 } & + & - & \\
\hline+ & 12 & 1 & 13 \\
- & 0 & 8 & 8 \\
\hline Total & 12 & 9 & 21 \\
\hline
\end{tabular}

Teste de McNemar: os resultados dispensam análises.
Tabela 8 Relação S/R do quadrante lateral (região R1) na mama esquerda detectada pela BS e pela BC.

\begin{tabular}{|c|c|c|}
\hline Paciente & BS & BC \\
\hline 1 & 125,00 & 85,00 \\
2 & 142,00 & 132,00 \\
3 & 40,00 & 20,35 \\
4 & 38,33 & 36,11 \\
5 & 13,00 & 3,06 \\
6 & 16,67 & 15,16 \\
7 & 22,00 & 27,90 \\
8 & 10,00 & 2,98 \\
9 & 43,40 & 17,30 \\
10 & 19,00 & 4,28 \\
11 & 8,67 & 7,46 \\
12 & 17,00 & 12,19 \\
13 & 8,75 & 3,05 \\
14 & 64,00 & 11,06 \\
15 & 8,66 & 0,56 \\
16 & 9,00 & 0,91 \\
17 & 13,00 & 5,25 \\
18 & 11,10 & 12,15 \\
19 & 35,27 & 7,91 \\
20 & 116,00 & 45,00 \\
21 & 4,25 & 1,70 \\
\hline Média & 36,43 & 21,73 \\
Mediana & 17,00 & 11,06 \\
\hline & Teste de Wilcoxon: \\
& & T calc. $=10,00 * ;$ \\
BS crit. $=59,00$. \\
BC. \\
\end{tabular}

Tabela 11 Pacientes portadoras de próteses na mama esquerda, segundo a presença ou não de irregularidade de contornos (boceladura, deformidade) detectada pela BC e pela BS.

\begin{tabular}{|l|c|c|c|}
\hline \multirow{2}{*}{ BS } & \multicolumn{2}{|c|}{ BC } & \multirow{2}{*}{ Total } \\
\cline { 2 - 3 } & Regular & Irregular & \\
\hline Regular & 9 & 0 & 9 \\
Irregular & 2 & 10 & 12 \\
\hline Total & 11 & 10 & 21 \\
\hline
\end{tabular}

Teste de McNemar: $p=0,2500$ ou 25\%; porcentagem de concordância: $19 / 21=90,47 \%$.

Tabela 14 Pacientes portadoras de próteses na mama direita, segundo a presença ou não do sinal do "linguine" detectada pela BC e pela BS

\begin{tabular}{|c|c|c|c|}
\hline \multirow{2}{*}{ BS } & \multicolumn{2}{|c|}{ BC } & \multirow{2}{*}{ Total } \\
\cline { 2 - 3 } & + & - & \\
\hline+ & 3 & 0 & 3 \\
- & 0 & 19 & 19 \\
\hline Total & 3 & 19 & 22 \\
\hline
\end{tabular}

Teste de McNemar: os resultados dispensam análises. Porcentagem de concordância: $22 / 22=100 \%$.
Tabela 9 Relação $S / R$ da região retroareolar (região R3) na mama esquerda detectada pela BS e pela BC.

\begin{tabular}{|c|c|c|}
\hline Paciente & BS & BC \\
\hline 1 & 48,00 & 40,77 \\
2 & 12,00 & 26,00 \\
3 & 32,50 & 20,76 \\
4 & 55,00 & 22,56 \\
5 & 11,00 & 6,42 \\
6 & 7,00 & 1,37 \\
7 & 15,67 & 25,60 \\
8 & 8,13 & 8,80 \\
9 & 44,70 & 10,70 \\
10 & 10,00 & 2,37 \\
11 & 1,67 & 7,03 \\
12 & 9,60 & 5,90 \\
13 & 4,75 & 1,39 \\
14 & 36,00 & 18,33 \\
15 & 4,00 & 4,38 \\
16 & 17,75 & 8,02 \\
17 & 6,50 & 0,57 \\
18 & 1,90 & 3,48 \\
19 & 35,36 & 8,50 \\
20 & 12,00 & 62,06 \\
21 & 14,00 & 11,00 \\
\hline Média & 18,45 & 14,06 \\
Mediana & 12,00 & 8,10 \\
\hline & Teste de Wilcoxon: \\
& & $=59,60$ \\
\hline
\end{tabular}

T calc. $=64,00 ;$ T crit. $=59,00$

Tabela 12 Pacientes portadoras de próteses na mama direita, segundo a presença ou não de dobras do envelope elastomérico detectada pela $B C$ e pela $B S$.

\begin{tabular}{|c|c|c|c|}
\hline \multirow{2}{*}{ BS } & \multicolumn{2}{|c|}{ BC } & \multirow{2}{*}{ Total } \\
\cline { 2 - 3 } & + & - & \\
\hline+ & 12 & 4 & 16 \\
- & 0 & 6 & 6 \\
\hline Total & 12 & 10 & 22 \\
\hline
\end{tabular}

Teste de McNemar: $p=0,0625$ ou 6,25\%; porcentagem de concordância: $18 / 22=81,81 \%$.

Tabela 15 Pacientes portadoras de próteses na mama esquerda, segundo a presença ou não do sinal do "linguine" detectada pela BC e pela BS

\begin{tabular}{|c|c|c|c|}
\hline \multirow{2}{*}{ BS } & \multicolumn{2}{|c|}{ BC } & \multirow{2}{*}{ Total } \\
\cline { 2 - 3 } & + & - & \\
\hline+ & 1 & 1 & 2 \\
- & 0 & 19 & 19 \\
\hline Total & 1 & 20 & 21 \\
\hline
\end{tabular}

Teste de McNemar: os resultados dispensam análises. 
Na região $\mathrm{R} 3$ esquerda, 33,33\% da amostra apresentaram relação S/R da BS menor que da BC. Dois casos apresentaram artefato de maior brilho da mama esquerda em relação à contralateral, que foi gerado pelo fato de o braço das pacientes ter estado em íntimo contato com partes da bobina. Este tipo de alteração não mais foi encontrado após corrigir o posicionamento.

Sinha et al. ${ }^{(6)}$ encontraram relação $\mathrm{S} / \mathrm{R}$ maior com as BSs do que com as BCs, embora relatem exames feitos com $\mathrm{BCs}$ em 64 pacientes, com dupla bobina de ombro em 60 pacientes, com uma única bobina de ombro em 20 pacientes e com BS circular para uso variado em dez pacientes; essas pacientes não são as mesmas para fins de comparação pacientepaciente e apresentam dados numéricos da relação $\mathrm{S} / \mathrm{R}$ numa tabela de apenas duas pacientes.

Deformidade de contornos ou boceladuras são aspectos relacionados à localização da prótese, retropeitoral ou não, e também à contratura capsular. Irregularidade dos contornos é encontrada em próteses com algum grau de calcificação da cápsula fibrosa (Figura 2). Em certos casos pode estar associado a ruptura e apresentar-se-á com outros sinais concomitantes ${ }^{(\mathbf{8 , 1 1})}$.

No presente estudo, houve porcentagem alta de concordância: 90,47\% para o lado esquerdo e $81,81 \%$ para o direito. Com relação à direita, verificou-se que em $18,18 \%$ foram detectadas alterações pela BS que não o foram pela $B C$. No lado esquerdo, este valor caiu pela metade: $9,5 \%$. O teste de McNemar mostrou concordância significante entre os resultados das $\mathrm{BCs}$ e das $\mathrm{BSs}$, evidenciando que não há diferenças estatísticas ao utilizar-se esses métodos.

Dobras existem em muitos dos casos que possuam contratura capsular importante e é uma resposta da modelagem da prótese às forças físicas de pressão atuando sobre o elastômero. Em raras ocasiões, podem apresentar-se muito irregulares, proeminentes e podem ser confundidas com o sinal do "linguine"

A capacidade de detecção de dobras do elastômero pelas BCs e pelas BSs foram excelentes. Não houve nenhum caso detectado pela BC que não pudesse ser identificado pela $\mathrm{BS}$ em ambos os lados (Figura 3). Em cerca de $18 \%$ à direita e em $4,8 \%$ à esquerda, as dobras puderam ser caracterizadas pela BS e não foram visibilizadas pela $\mathrm{BC}$.

Sinha et al. ${ }^{(6)}$ relataram que a principal limitação da BC é que sua relação $\mathrm{S} /$ $\mathrm{R}$ não é eficaz para uma boa visibilização de detalhes relevantes, tais como ruptura intracapsular da prótese de silicone. Os resultados desse trabalho não são concordantes para a detecção do "linguine".

Analisando-se os resultados dos dois métodos, quanto à presença do sinal do "linguine" (Figura 4), patognomônico de ruptura intracapsular, é fácil verificar que não houve diferença, em nenhum caso das próteses na mama direita, demonstrando que ambos os métodos são iguais em relação a este importante parâmetro. Na mama esquerda, dos 21 casos analisados, apenas um não foi concordante, o que não é estatisticamente significante. Esta paciente possuía prótese bilateral e a relação $\mathrm{S} / \mathrm{R}$ da mama esquerda era 3,5 vezes maior pela BS. A outra paciente com sinal do "linguine" à esquerda possuía relação $S / R$ 1,6 vez maior e foi concordante.

O conhecimento de que é possível detectar-se alterações nas próteses que permitam chegar a conclusões definitivas utilizando-se da BC vem auxiliarnos na possibilidade de sua utilização na eventualidade de não possuir no equipamento uma bobina dedicada à mama.

\section{CONCLUSÃO}

Comparando-se a utilização de BCs e BSs na realização de RM de mamas com próteses de silicone, concluímos que efetivamente a BS possui melhor relação S/R que a BC. Não existe diferença significante na visibilização dos contornos das próteses, das dobras e rupturas do elastômero em função do tipo de bobina utilizada.

Considerando que dobras são indicativo de integridade das próteses e o sinal de "linguine" é indicativo de ruptura intracapsular, e que as rupturas extracapsulares são facilmente diagnosticadas pela mamografia, método mais barato, postulamos que é válido usar a BC quando não se tem disponível a bobina específica para mamas na avaliação das próteses mamárias de silicone.

\section{REFERÊNCIAS}

1. Stewart NR, Monsees BS, Destouet JM, Rudloff MA. Mammographic appearance following implant removal. Radiology 1992;185:83-5.

2. Kessler DA. The basis of the FDA's decision on breast implants. N Engl J Med 1992;326:1713-5.

3. Theophelis LG, Stevenson TR. Radiographic evidence of breast implant rupture. Plast Reconstr Surg 1986;78:673-5.

4. Ahn CY, Shaw WW, Narayanan K, et al. Definitive diagnosis of breast implant rupture using magnetic resonance imaging. Plast Reconstr Surg 1993;92: 681-91.

5. Gorczyca DP, Sinha S, Ahn CY, et al. Silicone breast implants in vivo: MR imaging. Radiology 1992;185:407-10.

6. Sinha S, Gorczyca DP, DeBruhl ND, Shellock FG, Gausche VR, Bassett LW. MR imaging of silicone breast implants: comparison of different coil arrays. Radiology 1993;187:284-6.

7. Gorczyca DP, DeBruhl ND, Ahn CY, et al. Silicone breast implant ruptures in an animal model: comparison of mammography, MR imaging, US, and CT. Radiology 1994;190:227-32.

8. Mund DF, Farria DM, Gorczyca DP, et al. MR imaging of the breast in patients with silicone-gel implants: spectrum of findings. AJR 1993;161: 773-8.

9. Gorczyca DP, DeBruhl ND, Mund DF, Bassett LW. Linguine sign at MR imaging: does it represent the collapsed silicone implant shell? Radiology 1994;191:576-7.

10. Berg WA, Caskey CI, Hamper UM, et al. Singleand double- lumen silicone breast implant integrity: prospective evaluation of MR and US criteria. Radiology 1995;197:45-52.

11. Everson LI, Parantainen H, Detlie T, et al. Diagnosis of breast implant rupture: imaging findings and relative efficacies of imaging techniques. AJR 1994;163:57-60. 